\title{
Research Progress on the Relevance between Intestinal Flora and Colorectal Cancer
}

\author{
Yang Hui ${ }^{1 *}$, Junming $\mathrm{Hou}^{2}$, Yong $\mathrm{Jia}^{2}$, Ming Dong ${ }^{2}$, Feixue Feng ${ }^{2}$, Jing Jiang², Dezhen Yang ${ }^{2}$ \\ ${ }^{1}$ Shaanxi University of Chinese Medicine, Xixian District 712046, China. E-mail: tracywhiteh@163.com \\ ${ }^{2}$ Affiliated Hospital of Shaanxi University of Chinese Medicine, Xianyang 712000, China.
}

\begin{abstract}
Cancer is a common chronic disease all over the world, which will cause serious health burden. At present, the debate about the role of intestinal flora in the prevention and control of cancer has always existed. Therefore, researchers should pay close attention to the impact of intestinal flora on several cancers (such as colon cancer, liver cancer and breast cancer). In addition, it is reported that intestinal flora may also affect the efficacy of cancer chemotherapy and immunotherapy. This paper introduces some energy research results to help clear the relationship between intestinal flora and cancer, even cancer micro environment. It can help clarify the mist of cancer and gut microbiota, let those little creatures to serve the progress of improving mankind living condition and of health and medicine.
\end{abstract}

Keywords: Intestinal Flora; Colorectal Cancer; Anti-cancer; Mechanism of Cancer Micro Environment; Review

Foundings:

1. Preclinical study on the treatment of rectal cancer with Chinese medicine "Ehuang Enema"

2. Effects of Ehuang enema on intestinal flora and intestinal mucosal barrier function in rats with rectal cancer by high throughput sequencing

3. Study on the effect of aspirin on intestinal flora of rats by high throughput sequencing

\section{Introduction}

Colorectal cancer is one of the most common malignant tumors in the digestive tract and its etiology has not been fully elucidated ${ }^{[1]}$. Intestinal flora plays an important role in the pathogenesis of colorectal cancer, but its mechanism is unknown. The number of intestinal flora is 10 times that of human eukaryotic cells, 150 times that of the human genome, with a total of more than 100 trillion and more than 1000 species, of which anaerobic bacteria account for the vast majority ${ }^{[2]}$. Physiologically, the intestinal flora maintains relative homeostasis and interacts with the host. It plays an important role in nutrition, resisting pathogen invasion and promoting and maintaining normal immune function. When the intestinal flora is in equilibrium, the digestion, metabolism and immune system of the human body are in equilibrium. When the balance of intestinal flora is destroyed by various factors inside and outside the body and exceeds the regulating ability of the human body, it will affect the normal physiological function of the human body and lead to the occurrence of disease when it is serious. Intestinal microecology with the highest abundance and largest number of bacteria in the digestive tract is closely related to inflammatory bowel disease, colon polyps and colorectal cancer. Homeostasis is destroyed, which can lead to intestinal flora imbalance, and then induce colon cancer. But the mechanism is not

Copyright (C) 2020 Yang Hui et al.

doi: 10.18686/aem.v9i2.165

This is an open-access article distributed under the terms of the Creative Commons Attribution Non-Commercial License

(http://creativecommons.org/licenses/by-nc/4.0/), which permits unrestricted non-commercial use, distribution, and reproduction in any medium, provided the original work is properly cited. 
very clear. The main ones reported are: inducing chronic inflammation, synthesizing biotoxins to hinder intestinal epithelial cell cycle regulation, producing toxic metabolites, activating carcinogens such as heterocyclic amines. Research suggests that in the case of human intestinal flora disorder, some metabolic components cause inflammation by binding to receptors, which stimulates the secretion of inflammatory factors, and other reaction, leading to colon cancer.

The dominant bacteria in coliform are pseudomonas, snail, verruca microbe, clostridium, prevo, lactic acid bacteria, etc ${ }^{[3]}$. There are obvious differences in intestinal flora between patients with straight colon cancer and normal people. The current study found that the structure of intestinal flora in patients with colorectal cancer has changed greatly compared with those in health. Enterobacteria such as bacteroides, clostridium, bacteroides fragilis, enterococcus faecalis, streptococcus mutans and so on are associated with the development of colorectal cancer $^{[4]}$. Scanlan et al ${ }^{[5]}$ found that in colorectal cancer patients and adenomatous polyposis patients, the flora and their metabolites changed significantly, among which C. coccoides subgroups and clostridium leptum increased significantly. Sobhani et $a l^{[6]}$ found that bacteroides and prevotella in the feces of colorectal cancer patients were higher than those of healthy people. Tingting $\mathrm{Wang}^{[7]}$ and $\mathrm{Na} \mathrm{Wu}^{[8]}$ found that bacteroides, enterococcus, shigel, escherichia, streptococcus, klebsiella and pepso streptococcus were significantly increased in fecal flora of patients with colorectal cancer; However, the number of trichiaceae (Lach-nospiraceae), faecalibacterium and roseburia producing butyrate decreased significantly.

\section{Intestinal microflora can inhibit and promote the occurrence of colon cancer, which is mainly related to the specific types of microflora}

Probiotics such as lactobaci llus and bifidobacterium can inhibit the occurrence of colon cancer. Lactobacillus can induce the production of many cytokines, including IL-12, TNF - $\alpha$, etc ${ }^{[9]}$. The known physiological functions of lactobacilli are: to prevent the invasion and colonization of the intestinal tract by pathogenic bacteria, to maintain the micro ecological balance of the intestinal tract, to prevent and inhibit the occurrence of tumors, to enhance the immunity of the body, to promote digestion, to reduce cholesterol, to inhibit the production of endotoxin, to delay aging and anti radiation, etc. Bifidobacterium is the most numerous probiotics in the intestinal tract. Experiments show that bifidobacterium can enhance the expression of bax, which can induce the apoptosis of cancer cells and prevent the occurrence of colon cancer by down regulating the expression of bcl-2. In addition, as the ligand of toll like receptor, the phospholipid wall on its surface can activate the natural immune response and induce apoptosis of tumor cells, which has antitumor effect ${ }^{[10]}$.

As opposed to probiotics, harmful bacteria are a general term for bacteria that can cause disease in the host. The intestinal bacteria that cause colon cancer are streptococcus (streptococcus), enterococcus faecalis (ente-rococcus faecalis), enterotoxin producing bacteria (enteroto-xigenic bacteroides fragilis) and so on. Abdulamir et $a l^{[11]}$ found that after streptococcus infection in mice, the bacteria over expressed flagellin, which was beneficial to colonize the bacteria in tumor tissues, and the bacteria promoted the high expression of inflammatory-related signaling pathways in mouse intestinal epithelial cells, including the high expression of cyclooxygenase-2 (COX-2), which triggered intestinal inflammation. Through high-throughput sequencing technology, the fecal samples and intestinal cavity microbes of colon cancer patients and normal people were analyzed and compared, and it was found that the number of digestive streptococcus in the excreta of colon cancer patients increased compared with that of healthy people $e^{[12-15]}$.

Wang et $a l^{[16]}$ confirmed from animal experiments that some enterococcus faecalis can produce superoxide anion, which induces colon cancer. IL-10 knockout mice were infected with enterococcus faecalis which could produce superoxide anion in the experimental group and in the control group with enterococcus faecalis which did not produce superoxide anion IL-10 knockout mice. Experimental mice showed intestinal inflammation, DNA injury and colon cancer. The mice in the control 
group only caused enteritis and had no tumor. Enterococcus faecalis-induced colon cancer has been shown to be a diffusive chromosomal breaker (e.g. 4-hydroxy-2-non, 4-h by inducing mucosal macrophages ydroxynon-2-enal), which mediated DNA damage through bystander effects.

For example, bacteroides fragilis can secrete a kind of bacteroidin fragilis, which can cause DNA damage of colon epithelial cells. By activating the signal pathways of Wnt, NF- $\mathrm{K}$ B, STAT3, and so on, it can cause the inflammatory reaction of intestinal epithelium and promote colon cancer. The formation of intestinal microflora through the influence of inflammatory microenvironment leads to the imbalance of intestinal microflora in colon cancer, which leads to the conditional pathogenicity, the increase of mucus permeability, the migration of bacteria and the activation of innate immune system ${ }^{[17]}$. It can cause inflammatory cells to secrete a large number of inflammatory factors and form intestinal inflammation microenvironment together with toxic metabolites of bacteria. Long-term repeated intestinal inflammation is easy to induce mutation of intestinal epithelial cells with generating malignant proliferation, which causes tumors. At present, there are two theories about the promotion of inflammation by intestinal flora leading to colon cancer: alpha bug theory and driver passenger theory.

In alpha bug model, enterotoxin fragilis (ETBF) was used as an example. ETBF was implanted on the surface of colon mucosa to secrete fragile bacteroidin (BFT). It changed the structure of colon epithelial cells and mucosal immune function and finally led to the formation of colon cancer. It has been found that BFT can change the structure and function of colonic epithelial cells rapidly, including destroying tumor suppressor protein, e-cadherin and cadherin-e. These protein molecules can inhibit the formation of colonic tumors ${ }^{[18-19]}$. The decomposition of cadherin-e increases the permeability of colonic epithelial cells, which is the beginning of tumor development. Due to the release of cytokines, BFT can activate the transcription factor $\mathrm{NF}-\kappa \mathrm{B}$ and promote the synthesis and secretion of inflammatory cytokines by colonic epithelial cells. According to the theory of alpha bug, ETBF can directly lead to precancerous lesions of colon mucosa, and change the mucosal immune function and the structure of other intestinal flora can further promote the occurrence of colon cancer. Driver passenger model thinks that driver bacteria is the intestinal bacteria that may cause tumor, while passenger is the bacteria that has existed in the intestinal tract before. First of all, the bacteria in the intestinal driver cause caking the DNA damage of intestinal epithelial cells is the initial stage of colon cancer. Secondly, it induces the change of intestinal microenvironment. Passenger has a competitive advantage, surpassing driver in colon cancer and in quantity, and driver may disappear in tumor. According to the driver passenger theory, first of all, the colonization of driver bacteria in the colonic mucosa leads to the continuous inflammatory response of the mucosa, which leads to the precancerous lesions of the colonic mucosa by promoting the proliferation of colonic epithelial cells and producing DNA damage substances. With the accumulation of DNA damage material, the colonic epithelial cells mutated, and then changed from adenomatoid to adenocarcinoma. Whether probiotics can inhibit colon tumor tissue and whether passenger bacteria can promote the development of colon tumor tissue need further study. In a word, driver bacteria and passenger bacteria have different relations with colon tumor tissue and play different roles in the development of colon tumor.

Although some specific microflora will increase the risk of cancer, some beneficial intestinal bacteria can prevent various cancers by transforming phytochemicals into bioactive metabolites and fermenting dietary fiber into SCFAs, thus maintaining the integrity of intestinal mucosa and immune system. In addition, intestinal flora can affect the extragastrointestinal tissues by affecting the level of estrogen, that is, the risk of breast cancer. In addition, the potential mechanism of the interaction between beneficial flora and anticancer effect is still worth further exploration. On the other hand, harmful intestinal flora can be reduced or eliminated to maintain the homeostasis of intestinal flora. In general, intestinal flora may be an important mediator of diet cancer association, which suggests that we should go further to conduct the relationship between intestinal and cancers $^{[20]}$.

In the past, the study of intestinal microorganisms 
mainly depended on the traditional pure culture technology of microbial separation. When the method was used to restore the microflora in the laboratory, the amount of information obtained by anaerobic microorganisms was limited. In the past few years, researchers have studied the methods of fluorescence in situ hybridization, terminal restriction fragment length polymorphism (PCR), denaturing gradient gel electrophoresis (PGGE), biochip, and so on. For example, at a loss what to do with the detection of the dominant bacteria in the intestine, denaturing gradient gel electrophoresis can only detect the trace microorganisms, but the sensitivity is low. Fluorescence in situ hybridization technology must rely on the specificity of oligonucleotide probes, only detect the known bacteria but not identify the unknown microorganisms. It can verify the known, can not explore the unknown, and has defects in the judgment of microbial abundance. With the rapid development of detection technology and the maturity and promotion of the second generation sequencing technology, at present, high-throughput sequencing technology is mainly used to detect intestinal microorganisms. High throughput sequencing technology is a revolutionary change of traditional sequencing. It can sequence hundreds of thousands to millions of DNA molecules at a time. At the same time, high throughput sequencing makes it possible to analyze the transcriptome and genome of a species in a detailed and comprehensive way, so it is also called deep sequencing. High throughput sequencing technology, with its characteristics of large data volume, low cost and fast speed, has made a qualitative leap in the study of intestinal microbial metagenomics. It can more accurately and deeply analyze the microbial structure, composition, gene function, metabolic pathway and the impact of drugs on intestinal microorganisms.

\section{References}

1. Ahmedin J, Freddie B, Melissa MC, et al. Global cancer statistics. CA: A Cancer Journal for Clinicians 1900; 61(2): 69-90. doi: 10.3322/caac. 20107

2. Lozupone CA, Stombaugh JI, Gordon JI, et al. Diversity, stability and resilience of the human gut microbiota. Nature 2012; 489(7415): 220-30. doi: 10.1038 /nature 11550

3. Zhan H. The relationship between intestinal flora and colorectal cancer (in Chinese). Chinese Cancer Clinic 2015; 42(13): 675-679.

4. Ulger TN, Yagci A, Gulluoglu BM, et al. A possible role of bacteroides fragilis enterotoxin in the aetiology of colorectal cancer. Clinical Microbiology and Infection 2006; 12(8): 782-786. doi: 10.1111/j.1469-0691.2006.01494.x.

5. Pauline DS, Fergus S, Yvonne C, et al. Culture-independent analysis of the gut microbiota in colorectal cancer and polyposis. Environmental Microbiology 2008; 10(3): 789-798. doi: 10.1111/j.1462-2920.2007.01503.x.

6. Sobhani I, Tap J, Roudot TF, et al. Microbial dysbiosis in colorectal cancer (CRC) patients. PloS one 2011; 6(1): el6393. doi: 10.1371/journal.pone.0016393.

7. Wang T, Cai G, Qiu Y, et al. Structural segregation of gut microbiota between colorectal cancer patients and healthy volunteers. Isme Journal 2012; 6(2): 320-329. doi: 10.1038/ismej.2011.109.

8. Wu N, Yang X, Zhang R, et al. Dysbiosis signature of fecal microbiota in colorectal cancer patients. Microbial Ecology 2013; 66(2): 462-470. doi: 10.1007/s00248-013-0245-9.

9. Takeda K, Suzuki T, Shimada SI, et al. Interleukin-12 is involved in the enhancement of human natural killer cell activity by lactobacillus casei shirota. Clinical and Experimental Immunology 2006; 146(1): 109-115. doi: 10.1111/j.1365-2249.2006.03165.x.

10. Frick JS, Autenrieth IB. The gut microflora and its variety of roles in health and disease. Current Topics in Microbiology \& Immunology 2013; 358: 273-289. doi: 10.1007/82 2012217.

11. Liu M, Zhang J, Chen S, et al. Effect of TLRs on lipoteichoic acid of bifidobacterium inducing colon cancer cells apoptosis. Chinese Journal of Microecology 2008; 20(2): 116-119. doi: 10.3969/j.issn.1005-376X.2008.02.007.

12. Abdulamir AS, Hafidh RR, Bakar FA. Molecular detection, quan- tification, and isolation of streptococcus gallolyticus bacteria colonizing colorectal tumors: Inflammation-driven potential of carcino-genesis via IL-1, COX-2, and IL-8. Mol Cancer 2010; 9: 249.

13. Marchesi JR. Human distal gut microbiome. Environmental Microbiology 2011; 13(12): 3088-3102. doi: 10.1111/j.1462-2920.2011.02574.x.

14. Chen W, Liu F, Ling Z, et al. Human intestinal lumen and mucosa-associated microbiota in patients with colorectal cancer. PloS one 2012; 7(6): e39743. doi: 10.1371/journal.pone.0039743.

15. Wang $\mathrm{W}$, Chen $\mathrm{L}$, Zhou $\mathrm{R}$, et al. Increased proportions of bifidobacterium and the lactobacillus group and loss of butyrate-producing bacteria in inflammatory bowel disease. Journal of Clinical Microbiology 2013; 52(2): 398-406. doi: 10.1128/JCM.01500-13.

16. Ivanov $\mathrm{K}$, Kolev N, Tonev A, et al. Comparative 
analysis of prognostic significance of molecular markers of apoptosis with clinical stage and tumor differentiation in patients with colorectal cancer: a single institute experience. Hepato Gastroenterology 2009; 56(89): 94-98. doi: 10.1016/S0168-583X(98)00259-6.

17. Wu S, Rhee KJ, Zhang M, et al. Bacteroides fragilis toxin stimulates intestinal epithelial cell shedding and $\gamma$-secretase-dependent E-cadherin cleavage. Journal of Cell Science 2007; 120(Pt 11): 1944-1952. doi: 10.1242/jcs.03455.
18. Remacle AG, Shiryaev SA, Strongin AY. Distinct interactions with cellular e-cadherin of the two virulent metalloproteinases encoded by a bacteroides fragilis pathogenicity island. PloS one 2014; 9(11): e113896.

19. Tao J, Li S, Gan R, et al. Targeting gut microbiota with dietary components on cancer: Effects and potential mechanisms of action. Critical Reviews in Food Science \& Nutrition 2020; 60: 1025-1037. doi: 10.1080/10408398.2018.1555789. 